Artículo Original/ Original Article

http://dx.doi.org/10.18004/mem.iics/1812-9528/2020.018.02.47

\title{
Anticoagulación oral y riesgo de sangrado en pacientes con fibrilación auricular no valvular del Hospital Militar Central
}

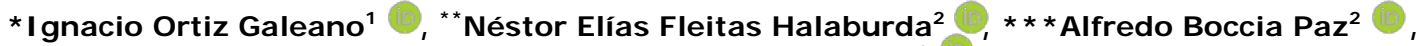 \\ $* * * *$ Néstor Arrúa Torreani ${ }^{2}$ \\ ${ }^{1}$ Universidad Nacional de Asunción. Facultad de Ciencias Médicas. Hospital de Clínicas. \\ San Lorenzo, Paraguay \\ ${ }^{2}$ Hospital Militar Central. Servicio de Clínica Médica. Asunción, Paraguay
}

Cómo referenciar este artículo/ How to reference this article:

\begin{abstract}
Ortiz Galeano I, Fleitas Halaburda NE, Boccia Paz A, Arrúa Torreani N. Anticoagulación oral y riesgo de sangrado en pacientes con fibrilación auricular no valvular del Hospital Militar Central. Mem. Inst. Investig. Cienc. Salud. 2020; 18(2): 47-53
\end{abstract}

\begin{abstract}
RE S U ME N
La fibrilación auricular es la arritmia más frecuente y puede causar accidente cerebrovascular. El objetivo de este estudio fue determinar el tipo de anticoagulante oral recibido y el riesgo de sangrado en pacientes con fibrilación auricular no valvular del Hospital Militar Central. El estudio, descriptivo, retrospectivo de corte transversal, incluyó a pacientes adultos con diagnóstico de fibrilación auricular no valvular del Hospital Militar Central desde enero de 2015 a agosto de 2018. Se determinaron las variables demográficas, diagnóstico de fibrilación auricular valvular y no valvular, tipo de anticoagulación oral recibida, escala de ictus $\left(\mathrm{CHA}_{2} \mathrm{DS}_{2}-\mathrm{VASc}\right)$ y de hemorragia (HAS-BLED), control de anticoagulación con el INR y adherencia al tratamiento anticoagulante. Se incluyeron 220 pacientes con fibrilación auricular, 60\% presentó fibrilación auricular no valvular, edad media de $71 \pm 4,6$ años, y el 57,8\% fueron hombres. Recibieron anticoagulación oral $79 \%$ de los pacientes, de los cuales el $80 \%$ recibió anticoagulante anti vitamina $\mathrm{K}$. El promedio de puntos en la escala de $\mathrm{CHA}_{2} \mathrm{DS}_{2}$-VASc fue de 3,5 puntos y en la escala de HAS-BLED el promedio de puntos obtenidos fue de 1,9 y los factores de riesgo más comunes de sangrado fueron la HTA y edad mayor a 65 años. En este estudio se encontró que la mayoría de los pacientes con FANV fueron anticoagulados, tenían alta adherencia al tratamiento y buen control de la ancoagulación. El anticoagulante oral más utilizado fue el anti vitamina K, hubo riesgo intermedio para el sangrado y los factores de riesgo de sangrado más comunes fueron la hipertensión arterial y la edad >65 años.
\end{abstract}

Palabras clave: fibrilación auricular, anticoagulación, accidente cerebrovascular, hipertensión.

\section{Oral anticoagulation and bleeding risk in patients with non-valvular atrial fibrillation of the Central Military Hospital}

\footnotetext{
*Docente-Investigador. Facultad de Ciencias Médicas. Universidad Nacional de Asunción

** Médico residente de Clínica Médica

$* * *$ Hematólogo

$* * * *$ Coordinador Docente, Medicina Interna
}

Fecha de recepción: noviembre 2019. Fecha de aceptación: julio 2020

Autor correspondiente: I gnacio Ortiz Galeano. Universidad Nacional de Asunción. Facultad de Ciencias Médicas. Hospital de Clínicas. San Lorenzo, Paraguay. Coronel Zoilo Cantero y Laurel. San Lorenzo, Paraguay. Celular: +595981374722

Email: ignacioortizgaleano@yahoo.es. Google analysis: UA-124832356-1 
Atrial fibrillation is the most frequent arrhythmia and can cause stroke. The objective of this study was to determine the type of oral anticoagulant received and the risk of bleeding in patients with non-valvular atrial fibrillation of the Central Military Hospital. The descriptive, retrospective cross-sectional study, included adult patients with a diagnosis of non-valvular atrial fibrillation from the Central Military Hospital from January 2015 to August 2018. Demographic variables, diagnosis of valvular and nonvalvular atrial fibrillation, type of oral anticoagulation received, stroke scale (CHA2DS2-VASC), hemorrhage (HAS-BLED) scale, control of anticoagulation with the INR and adherence to anticoagulant treatment were determined. Two hundred and twenty patients with atrial fibrillation were included, $60 \%$ presented non-valvular atrial fibrillation, mean age of $71 \pm 4.6$ years and $57.8 \%$ were men. Seventy nine percent of the patients received oral anticoagulation, of which $80 \%$ received anti-vitamin $\mathrm{K}$ anticoagulant. The average point on the CHA2DS2-VASc scale was 3.5 point and on the HAS-BLED scale the average point obtained was of 1.9 while the most common risk factors for bleeding were AHT and age over 65 years. In this study, it was found that the majority of patients with FANV was anticoagulated, had a high adherence to treatment, and good control of ancoagulation. The most widely used oral anticoagulant was anti vitamin $\mathrm{K}$, intermediate risk for bleeding and risk factors for bleeding, the most common was arterial hypertension and age $>65$ years.

Keywords: atrial fibrillation, anticoagulation, stroke, hypertension.

\section{NTRODUCCIÓN}

La fibrilación auricular (FA) es la arritmia cardiaca sostenida más frecuente ${ }^{(1)}$. Su prevalencia es de 2 a $5 \%$ de la población general y aumenta con la edad ${ }^{(2,3)}$. Es frecuente en los pacientes mayores de 65 años ${ }^{(2)}$. Hasta el $90 \%$ de los casos, el paciente no es consciente de su existencia ${ }^{(4)}$ y la primera manifestación puede ser la complicación de la FA como el accidente cerebrovascular $(\mathrm{ACV})^{(5)}$.

En Paraguay existe poco estudio publicado sobre la frecuencia de la FA en la población adulta, en un estudio realizado en un hospital regional del país encontraron $11,8 \%$, siendo más frecuente en el sexo masculino ${ }^{(6)}$.

La Sociedad Europea de Cardiología clasifica la FA en reciente comienzo, paroxística, persistente, persistente de larga duración y permanente ${ }^{(7)}$. En la FA persistente debe considerarse la cardioversión química o eléctrica y en la FA permanente no se considera la cardioversión.

El riesgo de complicaciones no es diferente entre los episodios de FA paroxísticos y las formas persistentes de la arritmia, en presencia de factores de riesgo ${ }^{(8)}$ y solo el tratamiento antitrombótico ha demostrado ser capaz de reducir las complicaciones y muertes relacionadas con la $\mathrm{FA}^{(9)}$.

El riesgo de ACV asociado a fibrilación auricular no es homogéneo, está incrementado por la presencia de factores de riesgo aditivos incluyendo ACV previo, accidente isquémico transitorio (AIT), hipertensión arterial, edad avanzada y diabetes ${ }^{(10,11)}$.

La identificación de los factores de riesgo de ACV ha llevado a la publicación de diversos esquemas para estratificar los riesgos de ACV. El esquema, expresado con el acrónimo $\mathrm{CHA}_{2} \mathrm{DS}_{2}-\mathrm{VASc}$ (insuficiencia cardiaca, hipertensión, edad $>75$ [valor doble], diabetes mellitus, ACV [valor doble], enfermedad vascular, edad 65 -74 años, y categoría de sexo [femenino]) ${ }^{(12)}$, amplía el esquema inicial $\mathrm{CHADS}_{2}$ (insuficiencia cardiaca, hipertensión, edad $>75$, diabetes mellitus, ACV [valor doble] $)^{(1)}$, considerando factores de riesgo adicionales de ACV que pueden influir en la decisión de la anticoagulación de los pacientes con $\mathrm{FA}^{(13)}$.

A pesar de contar con una terapia de anticoagulación oral eficaz establecida, los pacientes con FA y alto riesgo de presentar ictus son insuficientemente tratados ${ }^{(14)}$. EI enfoque para tromboprofilaxis en la FA requiere no solo estimar el riesgo de ACV, sino también considerar el riesgo de sangrado( ${ }^{(15)}$. El HAS-BLED score (del inglés: hipertensión no controlada, disfunción renal/hepática, ictus, historia o predisposición de sangrado, International Normalized Radio [INR] lábil, edad > 65 años, consumo de alcohol/drogas) ha sido propuesto para evaluar el riesgo de sangrado ${ }^{(16)}$. 
En el país hasta donde se conoce no existe estudio que evalúa el tipo de anticoagulante más usado en los pacientes con FA, la adherencia al tratamiento anticoagulante, el riesgo de sangrado en pacientes anticoagulados y los factores de riesgo para el sangrado. Conocer estas informaciones ayudaría a los médicos a optimizar el tratamiento anticoagulante y evitar las complicaciones de sagrado en los pacientes con FA.

El objetivo de este estudio fue determinar el tipo de anticoagulante oral recibido y el riesgo de sangrado en pacientes con fibrilación auricular no valvular del Hospital Militar Central.

\section{MATERI AL Y MÉTODO}

Se aplicó un diseño observacional, descriptivo, retrospectivo de corte transversal.

La población de estudio estuvo constituida por los pacientes internados en el Servicio de Clínica Médica del Hospital Militar Central desde enero del 2015 a agosto del 2017.

Los criterios de inclusión fueron pacientes mayores de 18 años, de ambos sexo con diagnóstico de FA de etiología valvular (FAV) y no valvular (FANV). Se excluyeron pacientes con diagnóstico de FA con fichas incompletas. Se utilizó un muestreo por conveniencia.

Se midieron variables demográficas (edad, sexo), diagnóstico de FAV y FANV (realizados por la clínica, electrocardiograma y ecocardiografía), anticoagulante oral (ACO) recibida para la FA: antagonistas de la vitamina $K$ (AVK) o con los nuevos anticoagulantes orales (NACO) y las que recibieron: doble antiagregación plaquetaria), escala de valoración de riesgo de ictus $\left(\mathrm{CHA}_{2} \mathrm{DS}_{2}-\mathrm{VASc}\right)$ en bajo riesgo: 0 punto, riesgo moderado: 1 punto, riesgo altæ2 puntos; escala de valoración de riesgo hemorrágico (HAS-BLED) en bajo riesgo: 0-2 puntos, alto riesgo: $\geq 3$ puntos), control de la anticoagulación con el INR (Ratio Internacional Normalizado) en rango terapéutico (INR) y en rango no terapéutico (INR) y la adherencia al tratamiento farmacológico anticoagulante (SI-NO).

Para el reclutamiento se solicitó permiso a las autoridades del Hospital Militar Central. Las variables fueron extraídas de los expedientes médicos de los pacientes. Luego fueron cargadas a una planilla electrónica de Excel ${ }^{\circledR}$. Las variables cualitativas se expresarán en frecuencias y porcentajes, las cuantitativas en medias y desviación estándar, utilizando el programa estadístico EpiDat 3.1 ${ }^{\circledR}$.

Para el cálculo de tamaño de muestra se utilizó el programa estadístico EpiDat 3. $1^{\circledR}$. Para una población de 220 pacientes con diagnóstico de FA en el periodo de estudio, frecuencia esperada de $12 \%{ }^{(6)}$, para una precisión $1 \%$, IC $95 \%$, el tamaño mínimo a incluir será de 209 sujetos.

Aspectos éticos: se respetó la confidencialidad de los datos personales. Los autores declaran que no reciben financiación externa ni tienen conflictos de interés comercial.

\section{RESULTADOS}

De un total de 2246 pacientes internados en el Servicio del Clínica Médica del Hospital Militar Central en el periodo de estudio presentaron FA 220 pacientes $(9,8 \%)$, de los cuales $133(60 \%)$ fueron FANV. La edad media de los pacientes con FANV fue de $71 \pm 4,6$ años, 77(57,8\%) fueron hombres.

De los pacientes con FANV recibieron anticoagulación 105 (79\%) pacientes, de los cuales $84(80 \%)$ pacientes recibieron AVK y $21(20 \%)$ pacientes recibieron NACO. Los pacientes que no recibieron anticoagulación oral $28(21 \%)$ recibieron doble antiagregación plaquetaria. De los 84 pacientes tratados con AVK recibieron en su totalidad el Acenocumarol y se pudo registrar una tasa de adhesión en $55(65,4 \%)$ pacientes hasta los 3 meses posteriores al inicio del tratamiento. De los 55 pacientes adheridos al tratamiento con AVK se registró que $44(80 \%)$ pacientes presentaron un INR entre 2-3 hasta los 3 meses posteriores al inicio del tratamiento.

Los pacientes que recibieron doble antiagregación plaquetaria obtuvieron un promedio de 4,2 puntos en la escala de $\mathrm{CHA}_{2} \mathrm{DS}_{2}$-VASc y un promedio de 2,5 puntos en la escala de HAS-BLED. 
En los pacientes que recibieron anticoagulación, el promedio de puntos en la escala de $\mathrm{CHA}_{2} \mathrm{DS}_{2}$-VASc fue de 3,5 puntos, de los cuales los factores de riesgos más comunes fueron la HTA $77(73,3 \%)$, la edad entre 65-74 años $77(73,3 \%)$ y la insuficiencia cardiaca $42(40 \%)$ (Tabla 1$)$.

Tabla 1: Distribución de pacientes con fibrilación auricular no valvular según factores de riesgos utilizados en la escala de $\mathrm{CHA}_{2} \mathrm{DS}_{2}$-VASc ( $n$ 133)

\begin{tabular}{lcccc}
\hline $\begin{array}{l}\text { Factores de } \\
\text { riesgos }\end{array}$ & $\begin{array}{c}\mathbf{n}=\mathbf{1 3 3} \\
\mathbf{( \% )}\end{array}$ & $\begin{array}{c}\text { Anticoagulados } \\
\mathbf{n = 1 0 5}(\mathbf{\%})\end{array}$ & $\begin{array}{c}\text { No anticoagulados } \\
\mathbf{n = 2 8}(\mathbf{\%})\end{array}$ & $\mathbf{p}$ \\
\hline Insuficiencia cardiaca & $49(36,8)$ & $42(40)$ & $7(25)$ & $<0,001$ \\
HTA & $98(73,6)$ & $77(73,3)$ & $21(75)$ & $<0,001$ \\
Edad > 75 años & $56(42,1)$ & $28(26,6)$ & $28(100)$ & 0,850 \\
Diabetes Mellitus & $21(15,7)$ & $14(13,3)$ & $7(25)$ & 0,064 \\
ACV/AIT & $21(15,7)$ & $7(6,7)$ & 14 & 0,064 \\
Edad entre 65-74 & $77(57,8)$ & $77(73,3)$ & 0 & $<0,001$ \\
años & & &
\end{tabular}

En cuanto a la puntuación obtenida en la escala de $\mathrm{CHA}_{2} \mathrm{DS}_{2}-\mathrm{VASc}$ en pacientes con FANV el máximo puntaje alcanzado fue de 6 puntos en $14(10,5 \%)$ pacientes y el puntaje más frecuente fue 3 obtenido en $77(58 \%$ ) (Tabla 2$)$.

Tabla 2: Puntuación en la escalda $\mathrm{CHA}_{2} \mathrm{DS}_{2}$-VASc en pacientes con fibrilación no valvular internados en el Servicio de Clínica Médica del Hospital Militar Central (133) $\mathrm{CHA}_{2} \mathrm{DS}_{2}$-VASc: escala de valoración de riesgo de ictus.

\begin{tabular}{lllll}
\hline $\begin{array}{l}\text { Puntuación de } \\
\mathbf{C H A}_{\mathbf{2}} \mathbf{D S}_{2} \text {-VASc }\end{array}$ & $\mathbf{n}(\%)$ & $\begin{array}{l}\text { Anticoagulados } \\
\mathbf{n}(\%)\end{array}$ & $\begin{array}{l}\text { No } \\
\text { anticoagulados } \\
\mathbf{n}(\%)\end{array}$ & $\mathbf{p}$ \\
\hline 6 & $14(10,5)$ & $7(6,7)$ & $7(25)$ & 0,705 \\
4 & $35(26,3)$ & $21(20)$ & $14(50)$ & 0,151 \\
3 & $77(58)$ & $70(66,6)$ & $7(25)$ & $<0,001$ \\
2 & $7(5,2)$ & $7(6,7)$ & 0 & 0,001 \\
\hline Total & 133 & 105 & 28 & \\
\hline
\end{tabular}

$\mathrm{CHA}_{2} \mathrm{DS}_{2}$-VASc: escala de valoración de riesgo de ictus.

En los pacientes que recibieron anticoagulación, en la escala HAS-BLED, el promedio de puntos obtenidos fue de 1,9 y los factores de riesgo más comunes fueron la HTA en $70(66,6 \%)$ pacientes, edad mayor a 65 años en $70(66,6 \%)$ pacientes (Tabla 3).

Tabla 3: Distribución de pacientes con fibrilación auricular no valvular según factores de riesgos utilizados en la escala HAS-BLED ( $n$ 133)

\begin{tabular}{|c|c|c|c|c|}
\hline $\begin{array}{l}\text { Factores de } \\
\text { riesgos }\end{array}$ & $n=133(\%)$ & $\begin{array}{l}\text { Anticoagulados } \\
n=105(\%)\end{array}$ & $\begin{array}{l}\text { No } \\
\text { anticoagulados } \\
n=28(\%)\end{array}$ & $\mathbf{p}$ \\
\hline HTA & $98(73,6)$ & $70(66,6)$ & $28(100)$ & $<0,001$ \\
\hline $\begin{array}{l}\text { Edad }>65 \\
\text { años }\end{array}$ & $98(73,6)$ & $70(66,6)$ & $28(100)$ & $<0,001$ \\
\hline ACV previo & $21(15,7)$ & $7(6,7)$ & $14(50)$ & 0,064 \\
\hline
\end{tabular}

HAS-BLED: escala de valoración de riesgo hemorrágico. 
La puntuación obtenida en la escala de HAS-BLED de los pacientes con FANV, el puntaje más alto fue de 3 puntos para $21(15,8 \%)$ pacientes, y el puntaje más frecuente fue de 2 puntos, obtenido por $84(63,2 \%)$ pacientes (Tabla 4 ).

Tabla 4: Puntuación en la escala HAS-BLED en pacientes con fibrilación no valvular internados en el Servicio de Clínica Médica del Hospital Militar Central (n 133).

\begin{tabular}{lllll}
\hline $\begin{array}{l}\text { Puntuación de } \\
\text { HAS-BLED }\end{array}$ & $\mathbf{n}(\%)$ & $\begin{array}{l}\text { Anticoagulados } \\
\mathbf{n}(\%)\end{array}$ & $\begin{array}{l}\text { No } \\
\text { anticoagulados } \\
\mathbf{n}(\%)\end{array}$ & $\mathbf{p}$ \\
\hline 3 & $21(15,8)$ & $7(6,7)$ & $14(50)$ & 0,064 \\
2 & $84(63,2)$ & $70(66,6)$ & $14(50)$ & $<0,001$ \\
1 & $28(21)$ & $28(26,7)$ & 0 & $<0,001$ \\
Total & 133 & 105 & 28 & \\
\hline
\end{tabular}

HAS-BLED: escala de valoración de riesgo hemorrágico.

\section{DISCUSIÓN}

En este estudio se encontró que la mayoría de los pacientes con FANV fueron anticoagulados, el tipo de anticoagulante más utilizado fue el AVK y los factores de riesgo de sangrado más comunes fueron la HTA y la edad >65 años. La mayoría de los pacientes presentaron un riesgo intermedio de sangrado. Estudios realizados en otros países encontraron resultados similares a este trabajo ${ }^{(17,18)}$.

Gonzalez Galeano M (6) encontró una frecuencia mayor de FA en pacientes de características similares a este estudio en pacientes del Hospital Regional de Coronel Oviedo, en cambio Wojszel ZB, et al. ${ }^{(19)}$ una frecuencia superior en una población de mayor edad. Estudios revelan la mayor frecuencia de la FA con el aumento de la edad de las personas ${ }^{(2,3)}$.

La FANV se encontró en más de la mitad de los pacientes estudiados con FA, en España en un estudio realizado en una población, dos tercio de los pacientes con FA fueron FANV ${ }^{(20)}$. La mayor frecuencia de la FANV podría deberse a la presencia de factores de riesgo y enfermedad cardiovascular concomitante en los pacientes como la HTA y la cardiopatía isquémica ${ }^{(21)}$.

Más de dos tercios de los pacientes con FANV fueron anticoagulados y en su mayoría el tipo de anticoagulante utilizado fue AVK, el resto de los pacientes recibieron doble agregación plaquetaria. El AVK utilizado en su totalidad fue el acenocumarol. El motivo del mayor uso de AVK fue el costo, los NACO son más costosos que los AVK, dificultando el acceso prolongado a estos nuevos fármacos. Existe efectividad de los AVK con acenocumarol en pacientes con FANV, sin embargo, la incidencia de complicaciones hemorrágicas es mayor ${ }^{(18)}$. En Cuba en pacientes con fibrilación auricular no reumática encontraron insuficiente la anticoagulación oral ${ }^{(22)}$. Aguilera Alcaraz MB, et al. ${ }^{(17)}$ encontraron también que la mayoría de los pacientes con FA fueron anticoagulados, y el tipo de anticoagulante más utilizado en los pacientes fue el AVK, en cambio en el trabajo de la Figuera $M$, et al. ${ }^{(23)}$ en España, los NACO fueron los más utilizados como tratamiento anticoagulante, y en más de la mitad fue el rivaroxaban.

La taza de adherencia al tratamiento anticoagulante oral y el control de la anticoagulación fueron adecuada en los pacientes. En otros estudios la calidad de la anticogulación fue deficiente ${ }^{(20)}$, con controles sub óptimas ${ }^{(17)}$ pero con la adherencia elevada ${ }^{(23)}$.

En cuanto a la doble antiagregación, cuya utilización en nuestro medio va decreciendo debido al mejor manejo de los ACO con las escalas de estratificación del riesgo y las posibilidades de acceso a los controles de INR como hemos visto en los resultados obtenidos, es importante destacar que su elección se debió a factores socio-culturales de los pacientes con indicación de ACO y no por presentar un alto riesgo de sangrado, a pesar del conocimiento sobre su efectividad, la cual es muy inferior en la prevención del ACV isquémico en comparación con el tratamiento anticoagulante ${ }^{(24)}$. 
Los factores de riesgo de tromboembolia en los pacientes con FANV fueron la edad $>75$ años y la HTA. En Brasil encontraron como factores asociados de tromboembolia en pacientes de un hospital general la edad $>75$ años, sexo masculino, consumo de tabaco, HTA, diabetes, presencia de ateromas en aorta y carótidas, INR subóptima y mala adherencia al tratamiento ${ }^{(25)}$.

En este estudio se encontró que los factores de riesgo de sangrado más comunes fueron la HTA y la edad $>65$ años y que la mayoría de los pacientes tuvieron riesgo intermedio para el sangrado. En otros estudios los factores de riesgo de sangrado fueron el IMC bajo, la diabetes, la insuficiencia cardiaca izquierda y la edad $>75$ $\operatorname{años}^{(26,27)}$.

Este estudio se realizó en pacientes de un solo hospital y constituye una limitación por que dificulta la generalización de los resultados.

Los resultados obtenidos de este estudio son de utilidad para demostrar que en los pacientes con FANV se puede prevenir la tromboembolia con el uso los AVK y el riesgo de sangrado es bajo si se tiene en cuenta las características de cada paciente.

\section{Fuentes de financiamiento:}

Este trabajo ha sido financiado por los autores.

\section{Conflicto de interés}

Los autores declaran no tener conflictos de interés.

\section{REFERENCI AS BI BLI OGRÁFI CAS}

1. Deshpande S, Catanzaro J, Wann S. Atrial Fibrillation: Prevalence and Scope of the Problem. Card Electrophysiol Clin. marzo de $2014 ; 6(1): 1-4$.

2. Prevalencia de fibrilación auricular en pacientes hospitalizados por Medicina interna - Science Direct [Internet]. [citado 9 de junio de 2020]. Disponible en:

https://www.sciencedirect.com/science/ article/ pii/S0120563319301044

3. de Moraes ERFL, Cirenza C, Lopes RD, Carvalho AC, Guimaraes PO, Rodrigues $A A E$, et al. Prevalence of atrial fibrillation and stroke risk assessment based on telemedicine screening tools in a primary healthcare setting. Eur J Intern Med. septiembre de 2019;67:3641.

4. Witkowski M, Bissinger A, Grycewicz T, Lubinski A. Asymptomatic atrial fibrillation in patients with atrial fibrillation and implanted pacemaker. Int J Cardiol. 15 de enero de 2017;227:583-8.

5. Perera KS, Sharma M, Connolly SJ, Wang J, Gold MR, Hohnloser SH, et al. Stroke type and severity in patients with subclinical atrial fibrillation: An analysis from the Asymptomatic Atrial Fibrillation and Stroke Evaluation in Pacemaker Patients and the Atrial Fibrillation Reduction Atrial Pacing Trial (ASSERT). Am Heart J. 2018;201:1603.

6. Atrial fibrillation of Paraguay hospital interior [Internet]. [citado 9 de junio de 2020]. http://scielo.iics.una. py/scielo.php?pid= S2072-

$81742015000200005 \&$ script $=$ sci_arttex t\&tlng $=p t$

7. 2016 ESC Guidelines for the Management of Atrial Fibrillation Developed in Collaboration With EACTS - PubMed [Internet]. [citado 9 de junio de 2020]. Disponible en: https: //pubmed.ncbi. nlm.nih.gov/27567 408/?from_term $=2016+E S C+G u i d e l i n e$ $\mathrm{s}+$ for +the + management+of+atrial+fibr illation+developed+in+collaboration+wi th+EACTS\&from_pos $=1$

8. Kim YG, Shim J, Boo KY, Kim DY, Oh S$K$, Lee $K-N$, et al. Different influence of cardiac hemodynamics on thromboembolic events in patients with paroxysmal and non-paroxysmal atrial fibrillation. PloS One. 2019; 14(3): e0214743.

9. Cavallari I, Ruff CT, Nordio $F$, Deenadayalu N, Shi M, Lanz H, et al. Clinical events after interruption of anticoagulation in patients with atrial fibrillation: An analysis from the ENGAGE AF-TIMI 48 trial. Int J Cardiol. 15 de 2018; 257: 102-7.

10. Guerra García D, Valladares Carvajal F, Bernal Valladares E, Díaz Quiñones J. Factores de riesgo asociados a ictus cardioembólico en pacientes con fibrilación auricular no valvular. Rev Finlay. marzo de 2018; 8(1):9-17.

11. Mentias A, Shantha G, Adeola O, Barnes GD, Narasimhan B, Siontis KC, et al. Role of diabetes and insulin use in the risk of stroke and acute myocardial 
infarction in patients with atrial fibrillation: A Medicare analysis. Am Heart J. 2019; 214: 158-66.

12. Guidelines for the Management of Atrial Fibrillation: The Task Force for the Management of Atrial Fibrillation of the European Society of Cardiology (ESC) PubMed [Internet]. [citado 9 de junio de 2020]. Disponible en: https:// pubmed.ncbi.nlm.nih.gov/20802 247/

13. Gilyarov MY, Konstantinova EV, Trukhin Al. Current possibilities of anticoagulant therapy in patients with atrial fibrillation. Klinicist. 1 de enero de 2015; 9(3): 15-21.

14. Ogilvie IM, Newton $N$, Welner SA, Cowell W, Lip GYH. Underuse of oral anticoagulants in atrial fibrillation: a systematic review. Am J Med. julio de 2010; 123(7):638-645.e4.

15. Garcia DA, Lopes RD, Hylek EM. Newonset atrial fibrillation and warfarin initiation: high risk periods and implications for new antithrombotic drugs. Thromb Haemost. diciembre de 2010; 104(6): 1099-105.

16. Lip GYH, Andreotti F, Fauchier L, Huber $K$, Hylek E, Knight $E$, et al. Bleeding risk assessment and management in atrial fibrillation patients: a position document from the European Heart Rhythm Association, endorsed by the European Society of Cardiology Working Group on Thrombosis. Eur Eur Pacing Arrhythm Card Electrophysiol J Work Groups Card Pacing Arrhythm Card Cell Electrophysiol Eur Soc Cardiol. mayo de 2011; 13(5): 723-46.

17. Aguilera Alcaraz BM, Abellán Huerta J, Carbayo Herencia JA, Ariza Copado C, Hernández Menárguez $F$, Abellán Alemán J. Valoración del tratamiento anticoagulante en pacientes diagnosticados de fibrilación auricular no valvular en una zona básica de salud. Clínica E Investig En Arterioscler. 1 de marzo de 2018;30(2): 56-63.

18. Castro C, Bustos L, Ocampo R, Molina E, Cabrero P, Vergara $R$, et al. Efectividad y seguridad del tratamiento anticoagulante con acenocumarol en fibrilación auricular no valvular. Rev Médica Chile. octubre de 2017; 145(10) : 1243-51.

19. Wojszel ZB, Kasiukiewicz A, Swietek M, Swietek ML, Magnuszewski L. CHA2DS2-VASc score can guide the screening of atrial fibrillation - cross- sectional study in a geriatric ward. Clin Interv Aging. 2019; 14:879-87.

20. Mora-Llabata V, Dubois-Marqués D, Roldán-Torres I, Mateu-Navarro C, Sanz-García JJ, Moreno-Ballester V, et al. Prevalencia de fibrilación auricular y características de la fibrilación auricular no valvular en la población general. Registro AFINVA. Rev Colomb Cardiol. febrero de 2017;24(1): 26-33.

21. Etiología y prevención de la fibrilación auricular | Revista Española de Cardiología [Internet]. [citado 9 de junio de 2020]. Disponible en: https://www. revespcardiol.org/esetiologia-prevencion-fibrilacionauricular-articulo-S1131358716300085

22. Linares GA, Álvarez KV, Armas LAS. Utilización de la anticoagulación en fibrilación auricular no reumática para profilaxis de accidentes cerebrovasculares. Rev Cuba Med. 30 de junio de 2015;54(2): 129-38.

23. de la Figuera M, Cinza S, Marín N, Egocheaga I, Prieto MA. Perfil clínico de pacientes con fibrilación auricular tratados con anticoagulantes orales de acción directa atendidos en atención primaria. Estudio SILVER-AP. Aten Primaria. 1 de junio de 2018;50 (6): 359-67.

24. Lip GYH, Tse HF, Lane DA. Atrial fibrillation. Lancet Lond Engl. 18 de febrero de 2012; 379(9816): 648-61.

25. Guevara GG, Díaz LAC, Calzado GF, Marrero JG, Vega VM. Factores de riesgo en complicaciones tromboembólicas cerebrales de pacientes con fibrilación auricular permanente no valvular. Rev Inf Científica. 2019;98(1):77-87.

26. Shinohara M, Fujino $T$, Yao S, Yano K, Akitsu $\mathrm{K}$, Koike $\mathrm{H}$, et al. Assessment of the bleeding risk of anticoagulant treatment in non-severe frail octogenarians with atrial fibrillation. J Cardiol. 2019; 73(1):7-13.

27. Comparative Validation of a Novel Risk Score for Predicting Bleeding Risk in Anticoagulated Patients With Atrial Fibrillation: The HAS-BLED (Hypertension, Abnormal Renal/Liver Function, Stroke, Bleeding History or Predisposition, Labile INR, Elderly, Drugs/Alcohol Concomitantly) Score PubMed [Internet]. [citado 9 de junio de 2020]. Disponible en: https://pubmed.ncbi.nlm.nih.gov/21111 555/ 